\title{
Intravital imaging of CTLs killing islet cells in diabetic mice
}

\author{
Ken Coppieters, Natalie Amirian, and Matthias von Herrath
}

Type 1 Diabetes Center, The La Jolla Institute for Allergy and Immunology, La Jolla, California, USA.

\begin{abstract}
Type 1 diabetes (T1D) is caused by autoimmune destruction of the insulin-producing $\beta$ cells in the pancreatic islets, which are essentially mini-organs embedded in exocrine tissue. CTLs are considered to have a predominant role in the autoimmune destruction underlying T1D. Visualization of CTL-mediated killing of $\beta$ cells would provide new insight into the pathogenesis of T1D, but has been technically challenging to achieve. Here, we report our use of intravital 2-photon imaging in mice to visualize the dynamic behavior of a virally expanded, diabetogenic CTL population in the pancreas at cellular resolution. Following vascular arrest and extravasation, CTLs adopted a random motility pattern throughout the compact exocrine tissue and displayed unimpeded yet nonlinear migration between anatomically nearby islets. Upon antigen encounter within islets, a confined motility pattern was acquired that allowed the CTLs to scan the target cell surface. A minority of infiltrating CTLs subsequently arrested at the $\beta$ cell junction, while duration of stable CTL-target cell contact was on the order of hours. Slow-rate killing occurred in the sustained local presence of substantial numbers of effector cells. Collectively, these data portray the kinetics of CTL homing to and between antigenic target sites as a stochastic process at the sub-organ level and argue against a dominant influence of chemotactic gradients.
\end{abstract}

\section{Introduction}

Type 1 diabetes (T1D) is a disease of unknown etiology that is characterized by the autoimmune destruction of pancreatic $\beta$ cells. $\mathrm{CD}^{+} \mathrm{T}$ cells, the principal effectors in safeguarding against viral infection, constitute a predominant population within insulitic lesions from recent-onset patients (1). In combination with MHC class I hyperexpression on islet cells around disease onset, the key molecular requirements are thus fulfilled to allow for recognition and $\beta$ cell-directed cytotoxicity $(2,3)$. A unique study by Skowera et al. demonstrated that preproinsulinreactive $\mathrm{CD} 8^{+} \mathrm{T}$ cells from human $\mathrm{T} 1 \mathrm{D}$ patients exert cytotoxicity against islets in vitro, which serves as a direct indication of their pathologic relevance (4). The precise etiological event that causes aberrant CTL activation in T1D remains unknown, but numerous studies have suggested that viral infections may be involved. Viral particles have been detected within islets and peripheral blood of affected patients $(3,5-7)$. Recent work has also demonstrated an association between detection of enteroviruses in the blood and initiation of islet autoimmunity or progression to clinically overt disease $(8-10)$. It was therefore inferred that viral infections may precondition susceptible hosts to initiation or acceleration of islet autoimmunity.

The RIP-LCMV mouse constitutes a widely used CTL-dependent diabetes model. In this nonsusceptible strain, insulitis is induced by viral infection with lymphocytic choriomeningitis virus (LCMV) which, after viral clearance, leads to a redirected CTL response against the viral glycoprotein that is transgenically expressed on $\beta$ cells (11-13). This model has been instrumental in defining the molecular conditions that enable antiviral CTL to enter the pancreatic islets and inflict $\beta$ cell damage. Parallels to human disease include the overexpression of MHC class I on islets (14), the relative unimportance of B cells (15, 16), and the contribution from $T$ cell chemoattractants such as

Conflict of interest: The authors have declared that no conflict of interest exists. Citation for this article: J Clin Invest. 2012;122(1):119-131. doi:10.1172/JCI59285.
CXCL-10 $(17,18)$. The well-documented epitope hierarchy of this system and the availability of TCR-transgenic mouse lines enable detailed studies of CTL effector functionality during diabetes development.

While the kinetics of primary $\mathrm{CD}^{+} \mathrm{T}$ cell activation have been extensively studied in secondary lymphoid organs (19-22), knowledge on the features of their cytotoxic activity at effector sites remains fragmentary. Quantification of the efficacy of CTL killing in vivo and the motility parameters that characterize activated CTL within peripheral target tissues have been subjected to meticulous in silico modeling approaches (23). Our current understanding, however, is hampered by the relative lack of experimental data that describe the kinetic properties of CTL at target sites other than lymph nodes and spleen. Such data can be directly recorded via intravital imaging at cellular resolution, with 2-photon microscopy being the technique of choice. Exciting recent examples include the in vivo imaging of CTL reactivity against tumor cells (24-26) and during viral infection of the central nervous system $(27,28)$. Breart and coworkers determined that the killing of one tumor cell by an individual CTL in vivo requires an average of 6 hours of interaction time (26). This finding confirms modeling data suggesting that the duration of killing is the actual rate-limiting step during CTL cytotoxicity and illustrates how intravital imaging leads to better characterization of these parameters.

Imaging diabetogenic immune responses in vivo at cellular resolution within pancreatic islets has been a particularly elusive goal. The majority of work has focused on strategies to noninvasively quantify $\beta$ cell mass during natural disease progression, experimental therapy, and islet transplantation (29). In order to gain better access to pancreatic islets and image at cellular resolution, purified islet populations were transplanted into the anterior chamber of the eye (30). Although this approach has clear advantages in terms of noninvasive longitudinal follow-up, the ectopic engraftment of enzymatically purified, handpicked, and cultured islets in combination with the immune-privileged nature of the transfer site limit applicability in studying 
A

B6 mouse expressing LCMV-GP and GFP in the $\beta$ cells

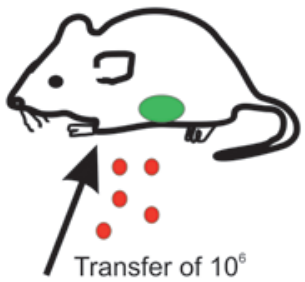

fluorescent $\mathrm{P} 14 \mathrm{~T}$ cells

B

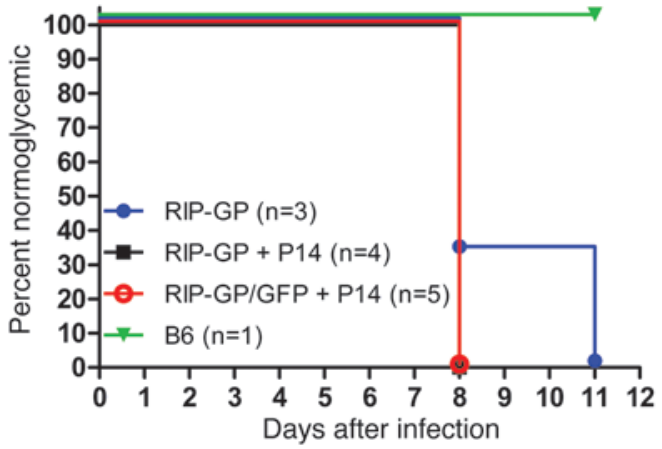

Day 7-8 after transfer: Imaging

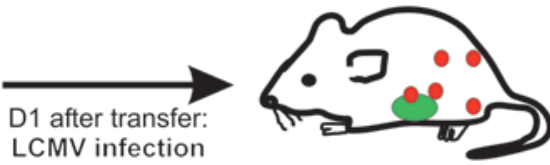

D
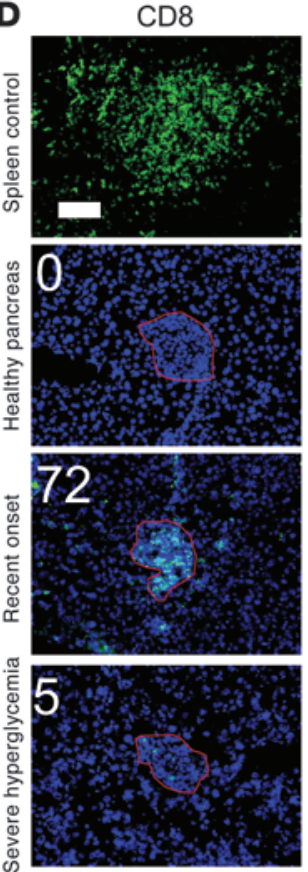

CD45.1
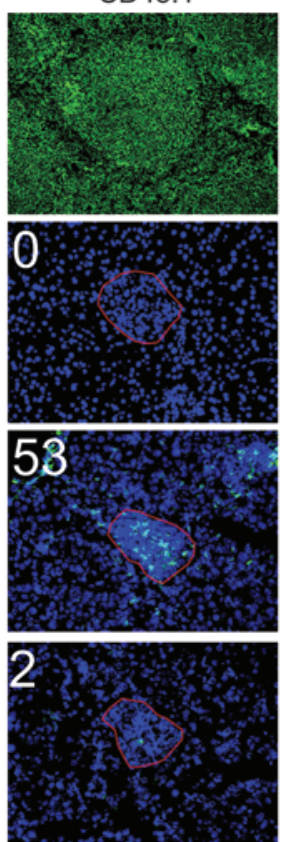

C

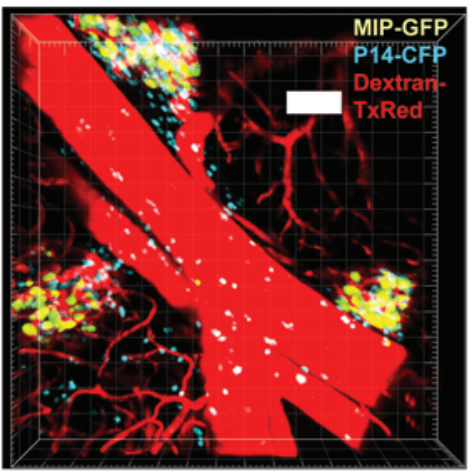

CD4
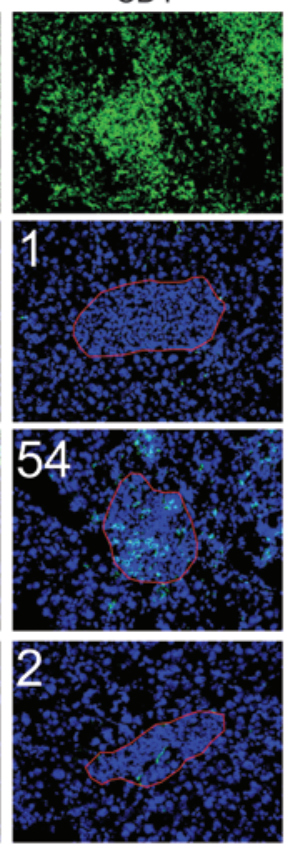

CD19
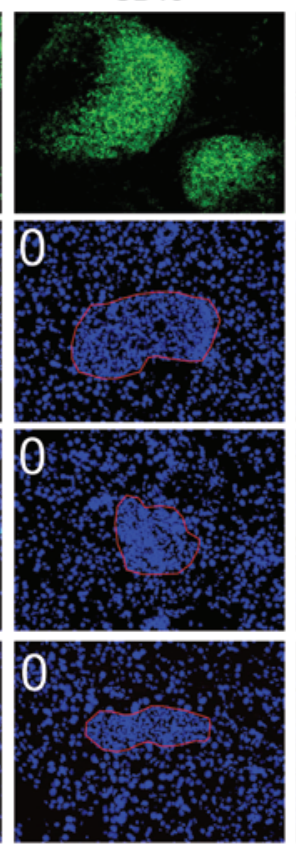

$\mathrm{F} 4 / 80$
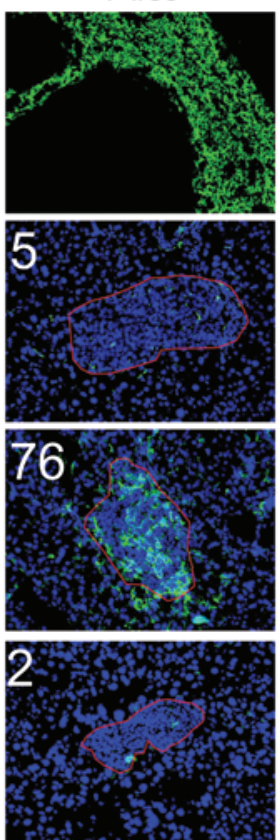

CD11c
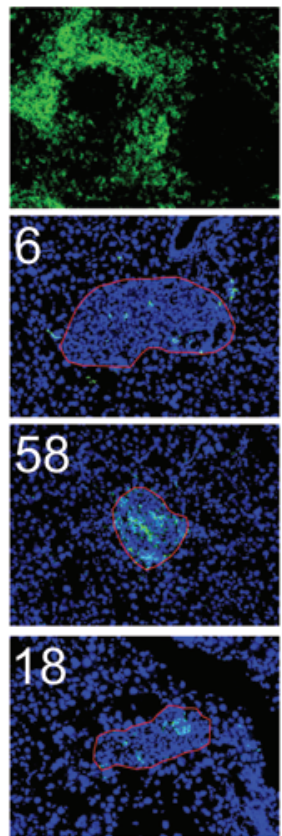

Figure 1

Establishment and characterization of a virus-induced diabetes model for in vivo pancreatic CTL imaging. (A) Recipient mice expressing GFP and viral antigen on their $\beta$ cells were injected with purified, naive, fluorescently labeled P14 CD8 ${ }^{+}$T cells 1 day prior to infection with LCMV. (B) Supplementation with TCR transgenic effectors results in a consistent synchronization of hyperglycemia onset as compared with the conventional model. (C) 2-Photon imaging was successful in capturing $\beta$ cell mass and infiltrating CTL ideally on days 7 and 8 after infection, immediately prior to diabetes onset. Vascular staining (here pseudocolored in red) reveals the local (micro-)vascular circuit. $w / h=1.52 / d=5 / z=41$. Scale bar: $100 \mu \mathrm{m}$. (D) Detailed immunofluorescent analysis of the cellular composition of the islet infiltrate at various stages of the diabetogenic process. Upper panel demonstrates validity of the stainings on frozen spleen sections. Transferred CD $8^{+} \mathrm{T}$ cells were detected by way of staining for the congenic marker CD45.1. Images represent consecutive sections of islets obtained at days 0,8 , and 10 after infection and are representative of 4 animals analyzed at each time point. Red outline defines approximate islet contours based on DAPI morphology for ease of interpretation. Scale bar: $100 \mu \mathrm{m}$. 

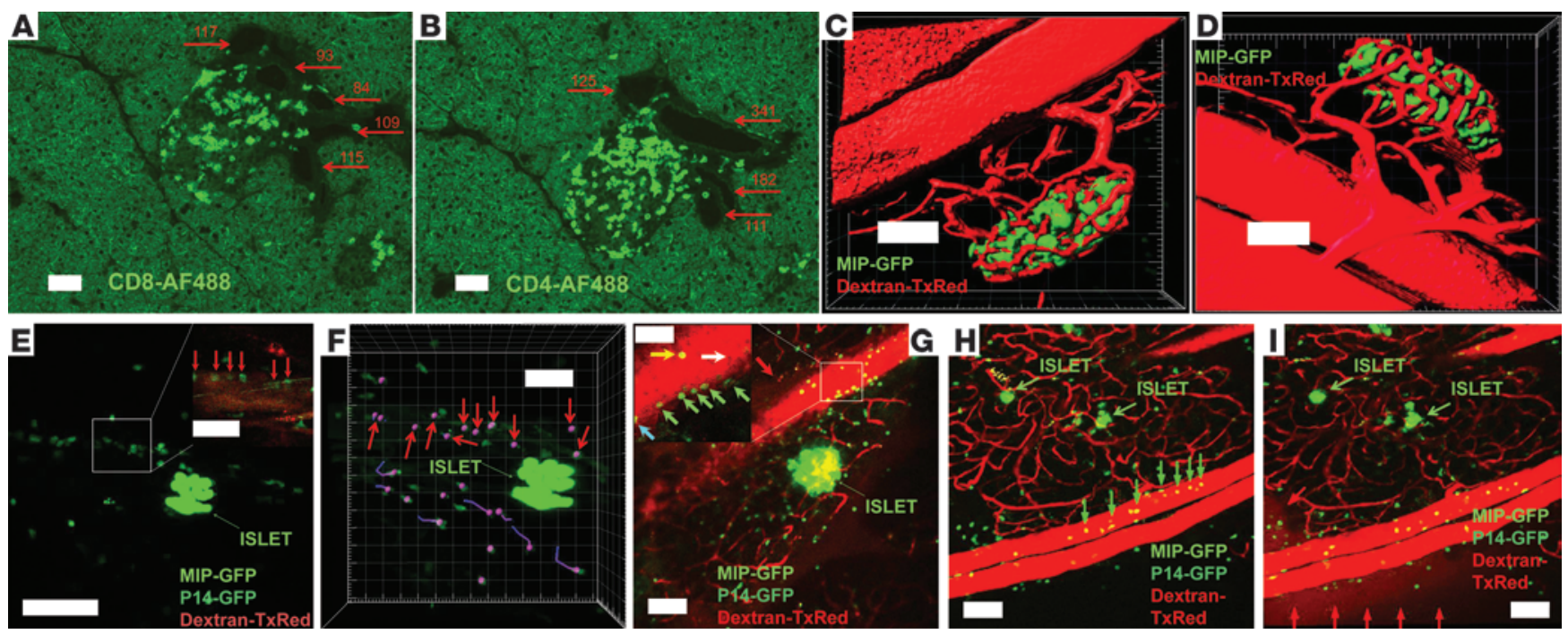

Figure 2

Diabetogenic CTL arrest in postcapillary pancreatic venules. (A and B) Pancreas sections from prediabetic RIP-LCMV.GP animals $(n=6)$ stained for CD8 and CD4. Autofluorescence shows general morphology. Red arrows indicate vascular structures with maximum diameter ( $\mu \mathrm{m})$. ( $\mathbf{C}$ and D; corresponds to Supplemental Video 1) Vascular staining in naive animals. $w / h=1.52 / d=5 / z=32$. (E) MIP image corresponds to Supplemental Video 2. $w / h=0.56 / d=4 / z=25$. CTL can be seen in virtual arrest within postcapillary venules (red arrows). Inset represents a single Z-plane. (F) Cellular tracking shows immotile cells in the vascular bed (red arrows), lacking the "dragontails" that signify motility. (G) MIP image corresponds to Supplemental Video 3. $w / h=1.52 / d=5 / z=21$. Setup analogous to $E$ in another prediabetic animal. Green arrows indicate arrested cells in contact with the vascular wall. Cyan arrows show cells "crawling" against the vascular wall. White arrow shows endogenous "ghost" cells in arrest against the endothelium. Yellow arrow shows freely flowing transferred cell. Red arrow shows leakage of the vascular dye. (H) MIP image corresponds to Supplemental Video 4. $w / h=1.52 / d=5 / z=26$. Setup analogous to $\mathbf{E}$ and $\mathbf{G}$ in another prediabetic animal. Green arrows point toward arrested transferred cells. (I) Image of the same region shown in $\mathbf{H} 8$ minutes after dye injection demonstrates vascular leakage of the dextran-Texas Red dye (red arrows). Scale bars: $100 \mu \mathrm{m}(\mathbf{A}-\mathbf{E}, \mathbf{G}-\mathbf{I}) ; 50 \mu \mathrm{m}(\mathbf{F}$, insets in $\mathbf{E}, \mathbf{G})$.

immune infiltration. To date, no study to our knowledge has been successful in visualizing the interactions of diabetogenic effectors with $\beta$ cells in a living animal at cellular resolution. This study applies a recently published and publicly accessible surgical technique for the exposure of the pancreas to allow for 2 -photon imaging at cellular resolution (31). We define here the kinetic features of CTL infiltration into pancreatic islets after viral infection and their interactions with $\beta$ cells that ultimately result in clinical hyperglycemia.

\section{Results}

Characterization of diabetogenic islet immunity in the RIP-LCMV.GP model after supplementation with naive TCR-transgenic $C D 8^{+} T$ cell effectors. Our aim was to visualize the kinetic behavior and fate of a population of diabetogenic CTLs within the context of the well-characterized RIP-LCMV.GP diabetes model. We previously designed a custom imaging technique that enables dynamic 2-photon imaging at cellular resolution in pancreatic islets from living animals in the presence of intact vascular circulation (31). While the latter technical report utilized a virus-free diabetes transfer model, the antigenic trigger employed in the present study was of viral origin (Figure 1A). Using a method analogous to that reported recently by Kang and coworkers (28), we used P14 TCR transgenic Thy $1.1^{+} \mathrm{CD}^{+} \mathrm{T}$ cells as a traceable representative of the CTL response (32). One day prior to infection, $1 \times 10^{6} \mathrm{MACS}$-purified, naive TCR-transgenic P14 cells were i.v. transferred to RIP-LCMV transgenic hosts (13), which express GFP under the mouse insulin promoter for imaging purposes (33). The P14 cells recognize the
GP33 epitope of the glycoprotein expressed by the LCMV, the same antigen that is transgenically expressed on the host's $\beta$ cells under the control of the insulin promoter. After activation, expansion, and subsequent viral clearance, endogenous effectors redirected to the pancreatic islets and generally caused hyperglycemia from 8 to 14 days after infection (Figure 1B). The transfer of P14 cells consistently resulted in a synchronization of diabetic symptoms, which is a desirable feature when the objective is to reproducibly monitor CTL effector motility and functionality in the presence of antigenic target, i.e., $\beta$ cells (discussed in ref. 31 ). This observation also demonstrates the active involvement of the transferred CTL population in diabetes induction. All imaging experiments outlined below were performed at day 7 or 8 after infection, immediately before or at the early onset of clinical hyperglycemia. During this narrow time window, pancreatic $\beta$ cells could be reliably imaged at cellular resolution, in conjunction with infiltrating CTL and (micro-)vascular staining (Figure 1C). In order for the P14 cells to remain visible after multiple rounds of proliferation, stable genetic labeling was required instead of conventional membrane dyes. We found that greatest imaging depth was achieved with GFP-labeled cells, followed by Cyan Fluorescent Protein (CFP), while DsRed was often not bright enough to yield sufficient detection (31).

Next, the local immunological islet milieu was defined in this model to allow for proper interpretation of the imaging data. Indeed, infiltration of the labeled cells of interest was accompanied by influx of endogenous leukocyte populations, which remained invisible for 2-photon detection. We therefore validated immunofluorescent staining protocols for various immune subset markers 


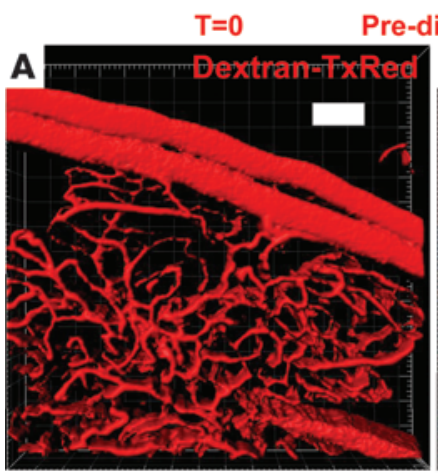

re-diabetic $\quad \mathrm{T}=10$
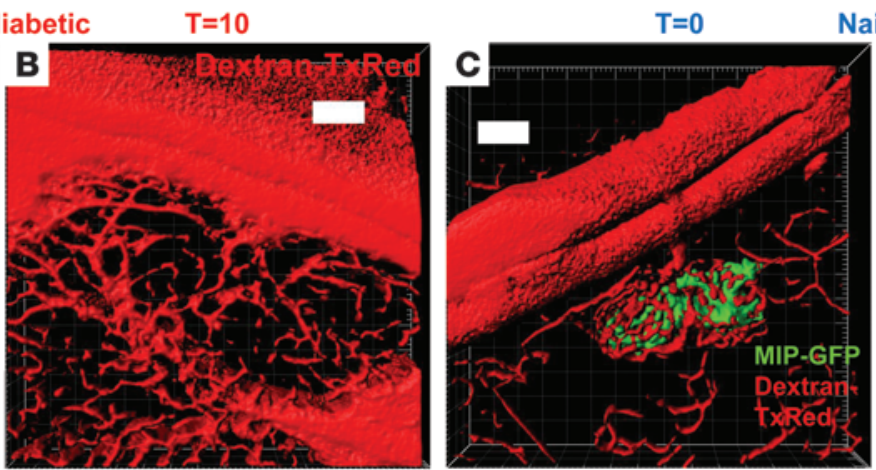

Naive $\quad \mathrm{T}=14$
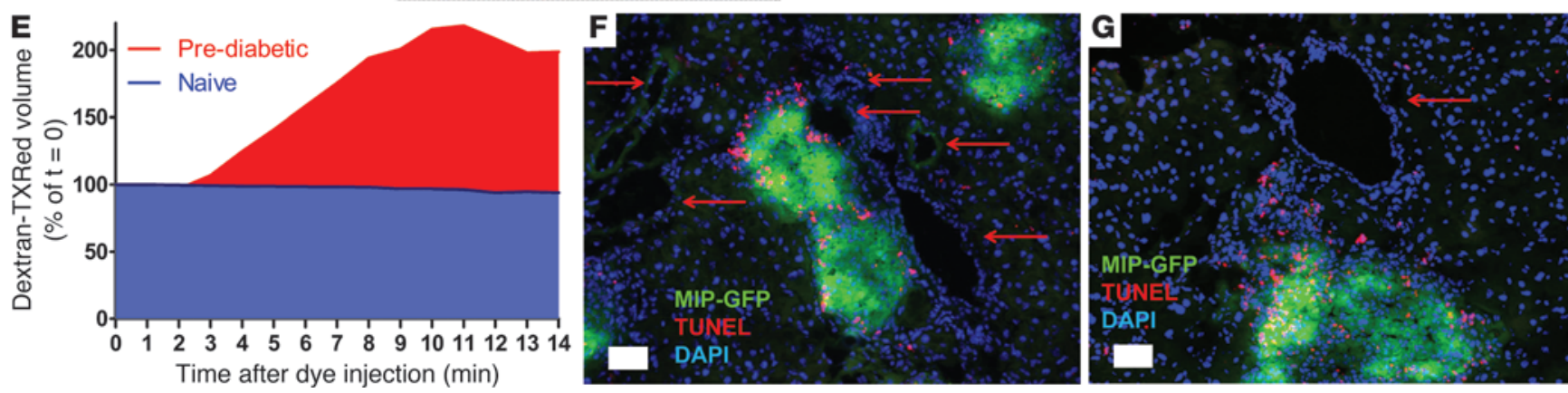

Figure 3

Profound leakiness of postcapillary islet venules is unrelated to endothelial apoptosis. (A and B) Isosurfacing rendition of vascular staining from region depicted in Figure 2, $\mathrm{H}$ and I, before and 10 minutes after injection of the dye, respectively. $w / h=1.52 / d=5 / z=26$. (C and $\mathbf{D}) \mathrm{Naive}$ control animal imaged before and 14 minutes after injection with dextran-Texas Red (dextran-TxRed). w/h $=1.52 / d=5 / z=33$. (E) Quantitative analysis of vascular leakiness as observed in $\mathbf{A}$ and $\mathbf{B}$ compared with baseline in $\mathbf{C}$ and $\mathbf{D}$. Leakiness was defined as increase in isosurfaced dextran-Texas Red-derived fluorescent signal and was normalized to $T=0$ immediately after injection. Representative of observations from 3 individual prediabetic animals and 3 controls. ( $F$ and $\mathbf{G}$ ) TUNEL staining was performed to reveal ongoing apoptosis in the transfer model during the late prediabetic phase. TUNEL+ nuclei are exclusively observed within the $\beta$ cell mass and inflammatory infiltrate. Red arrows indicate vascular structures and the lack of ongoing apoptosis within these regions. Images are representative of at least 3 consecutive sections from 4 individual prediabetic animals. Scale bars: $100 \mu \mathrm{m}$.

on frozen spleen sections (Figure 1D) and defined baseline levels in islets from naive, nondiabetic mice (Figure 1D). In agreement with common knowledge, no appreciable lymphocyte numbers were present, while the islets contained a small resident population of macrophages and dendritic cells. Around onset of hyperglycemia on days 7 through 8 (day 8 shown in Figure 1D), substantial numbers of $\mathrm{CD}^{+} \mathrm{T}$ cells had accumulated within the islet milieu, predominantly composed of transferred effectors, which were colabeled here with the congenic marker CD45.1. Considerable amounts of $\mathrm{CD}^{+} \mathrm{T}$ cells, macrophages, and dendritic cells were simultaneously present, but no B lymphocytes cells were observed. These data thus draw a detailed picture of the cellular milieu surrounding the transferred population of labeled CTLs at the time of imaging. From 1 to 2 days after development of full-blown diabetes (days 9-10 after infection), infiltration levels for all markers tested returned to baseline, apart from a few persistent cells (Figure 1D). This observation demonstrates the antigen-specific nature of islet infiltration and particularly leukocyte retention in this model and highlights that any $\mathrm{T}$ cell imaging in this model has to occur before antigen depletion.

Vascular CTL arrest in "leaky" postcapillary islet venules. Over years of routine immunohistochemical and immunofluorescent analysis of islet infiltration in the conventional RIP-LCMV.GP model, our lab consistently noticed that both $\mathrm{CD}^{+}$(Figure $2 \mathrm{~A}$ ) and $\mathrm{CD}^{+}$
(Figure 2B) lymphocytes appear to gain access to the islet milieu through noncapillary vessels directly adjacent to the islet site. Lymphocytes are often captured forming distinct migratory routes between such vascular structures and the infiltrate that gradually engulfs islets during the prediabetic phase. Intravital microscopy of CTL homing to nonlymphoid tissues offers the capacity to reveal lymphocyte interactions with the local vasculature. The imaging approach employed here enables deep-tissue $3 \mathrm{D}$ views of the tortuous microvascular network within intact islets (Figure 2, C and D, and corresponding Supplemental Video 1; supplemental material available online with this article; doi:10.1172/JCI59285DS1). An arteriole/venule pair with diameters approximately similar to the sectioned islet vessels in Figure 2, A and B, can be seen. This pair serves as supply and drainage sites, respectively, for the fine, tortuous network of capillaries that perfuse the islet cells.

In prediabetic mice supplanted with labeled CTLs, activated effectors were consistently found to interact with and stably arrest at the endothelial lining associated with postcapillary vessels (Figure 2, E-I, and corresponding Supplemental Videos 2 and 3). Cellular tracking showed arrest for at least 15 minutes (Figure $2 \mathrm{~F}$ ). In accordance with the general notion that the entire process of $\mathrm{T}$ cell diapedesis takes several hours $(34,35)$, not a single event of actual transendothelial migration was recorded in our studies. To our knowledge, the precise time line of CTL extravasation at auto- 
immune effector sites such as the prediabetic pancreas has never been evaluated in vivo, and we thus offer what we believe is the first direct indication that the temporal kinetics of this process may be in the range of hours.

Raw fluorescence data presented in Figure 2I show the decreased vascular confinement of injected dye and thus exhibit the "leaky" state of the vessels in which the CTL are found to arrest. In order to quantitatively assess the "leaky" vascular phenotype associated with the postcapillary venules in the prediabetic phase, we performed 3D isosurfacing analysis (Figure 3, A-D, and corresponding Supplemental Videos 4 and 5). In control animals, the aggregate volume of the local vascular network immediately after injection of vascular dye remained constant, in agreement with preserved vascular integrity and consequential confinement of the 70-kDa dye molecules (Figure 3, C-E, and Supplemental Video 5). In prediabetic animals, in contrast, the vascular dye was found to rapidly disperse in the surrounding acinar tissue, and the volume associated with fluorescence emanating from the injected dye more than doubled 10 minutes after administration (Figure 3, A, B, and E, and Supplemental Video 4). Given the observed CTL arrest at the vascular junction, we evaluated whether these interactions represented ongoing $\beta$ cell killing by the CTLs, as suggested by Savinov et al. (36). Extensive immunofluorescent TUNEL staining was therefore performed on frozen sections obtained from CTLsupplemented, infected mice to assess the frequency of apoptosis in the endothelial compartment (Figure 3, F and G). Whereas in this model, ample apoptosis was detected within the islet cell compartment and the associated infiltrate, no apoptosis was observed in the endothelial compartment. We therefore conclude that the highly permeable postcapillary islet venules in this model represent sites of CTL arrest as part of endothelial transmigration.

Diabetogenic CTLs migrate through exocrine tissue by random walk. $\mathrm{T}$ cell velocities in lymph nodes and spleen have been reported to be in the range of $10-15 \mu \mathrm{m} / \mathrm{min}$ (23), but no data exist on CTLassociated migration in the pancreas. Five representative time-lapse sequences were acquired (Figure 4, A-E, and corresponding Supplemental Videos 6 and 7) using different digital zoom factors. Distinct sequences contained variable amounts of $\beta$ cell-derived GFP signal (and thus antigen) and different degrees of infiltration. In these experiments, we specifically focused on the CTL population that migrates in direct proximity to, but not within, pancreatic islets, and we therefore used the brighter GFP P14 tag at the expense of $\beta$ cell resolution. In analogy to the mean track velocities reported in secondary lymphoid organs in the absence of antigen, it was found that acinar CTLs had associated velocities of approximately $10 \mu \mathrm{m} /$ min, with only results shown in Figure 4B deviating toward the 15 $\mu \mathrm{m} / \mathrm{min}$ range. As in lymphoid tissue, peak velocities higher than 25 $\mu \mathrm{m} / \mathrm{min}$ were recorded (37). Track straightness, also termed motility coefficient or meandering coefficient, was determined for the CTL population in each individual sequence and consistently averaged in the $0.4-0.5$ range. This parameter represents the ratio between the cell's total displacement from its original position and the length of its entire track and thus is a measure of cellular "restraint." These values are not absolute, but appear in the general range reported during CTL rejection of solid tumors (25) and antigen-specific T cells in inguinal lymph nodes (38). In the event of directional, unconfined migration, this value would approximate 1 , and therefore the measures reported here are a first indication that CTL migration in the acinar tissue lacks directionality. Arrest coefficients, which represent the percentages of time each cell migrates with instantaneous veloci- ties below an arbitrary value (here, $<3 \mu \mathrm{m} / \mathrm{min}$ ), were consistently low (within the 0.1-0.4 range). This parameter indicates that in the absence of direct interaction with viral antigen presented in an MHC class I context, cells rarely arrest for prolonged time periods. This also indirectly confirms that viral clearance at time of imaging is complete and suggests that the only cell population carrying antigen/MHC I complexes are the $\beta$ cells.

Arguably the best method to interpret motility patterns of entire cell populations is the displacement versus time $\mathrm{e}^{1 / 2} \mathrm{plot}(39)$. These plots are depicted for each population in Figure 4, A-E, and repeatedly show a linear relationship consistent with "Brownian" or random motility. Further, the slope of the line after regression can be used to calculate a motility coefficient that, except for sequence $\mathrm{B}$ (due to higher velocities), is in the $20-30 \mu \mathrm{m}^{2} / \mathrm{min}$ range. This measure has been experimentally reported to range from 50-100 $\mu \mathrm{m}^{2} / \mathrm{min}$ for $\mathrm{T}$ cells in lymphoid organs in the absence of antigen (23). This indicates that the combination of directionality of motion and cell velocity associated with CTL in the pancreas is substantially lower than in antigen-free lymphoid tissue. In conclusion, the migratory pattern associated with CTL in the prediabetic pancreas can be described as a random walk with relatively moderate motility and lacking cellular arrest.

Since pancreatic islets were reported to be a significant source of chemokines during diabetes development in mice (40) and humans (18), we asked whether a chemotactic component could be detected in CTL migration. We based our analysis on Okada et al. (41) and assumed that active chemotaxis would alter the behavior of CTLs as they migrate closer to the islets. Treating the entire population as a single entity would potentially disguise subtle chemotaxis, since cells far from the islet (and not/less under its presumed chemotactic influence) would compensate for cells located closer that do show directionality. The surrounding CTL population was therefore stratified according to distance from the islet centroid (Figure 4G). However, CTL in none of these regions showed net displacement toward the islet (Figure $4 \mathrm{H}$ ) or differences in track straightness (Figure 4I). Moreover, cells that migrated toward the islet showed similar track straightness compared with those that moved away (Figure 4J).

Finally, tracking results were compared using a noninfectious antigenic stimulus for CTL activation and expansion as reported previously (31). Briefly, instead of viral infection with LCMV, mice were treated with a combination of GP33 peptide and TLR-ligand adjuvants. After imaging and tracking, CTL motility patterns were found to be largely within the same range as when viral infection was employed (Figure 4F and corresponding Supplemental Video 8), displaying a clearly random walk. This finding suggests that strong peripheral CTL activation, possibly in conjunction with induction of vascular permeability, is sufficient to enable CTL to enter and migrate within the acinar tissue and indicates that signaling derived from local viral infection is not a requirement in this process.

Unimpeded yet nonlinear motility between anatomically adjacent islets. Since it was repeatedly observed in our imaging studies that CTLs could establish a 3D continuum between anatomically nearby islets (Figure 5, A and B, corresponding Supplemental Video 9), we wished to evaluate whether these cells follow defined, linear routes from one islet site to another via exocrine tissue. 3D regions of interest were drawn between adjacent islets, and cellular tracking was performed (Figure 5, C-E, and corresponding Supplemental Video 10). While it was found that these inter-islet regions exhib- 

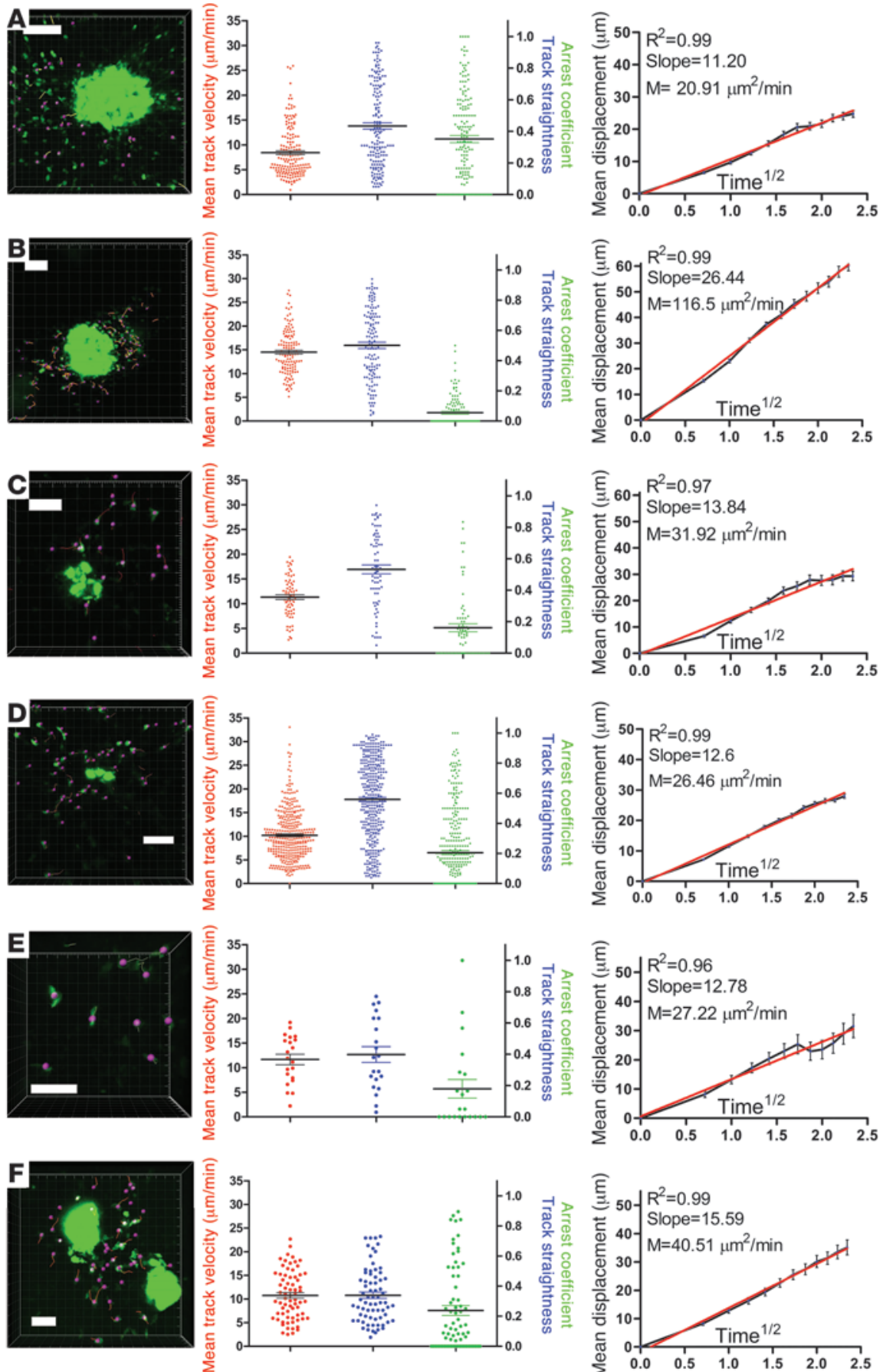
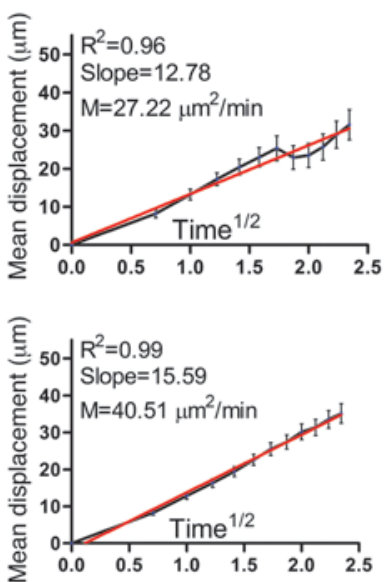

G
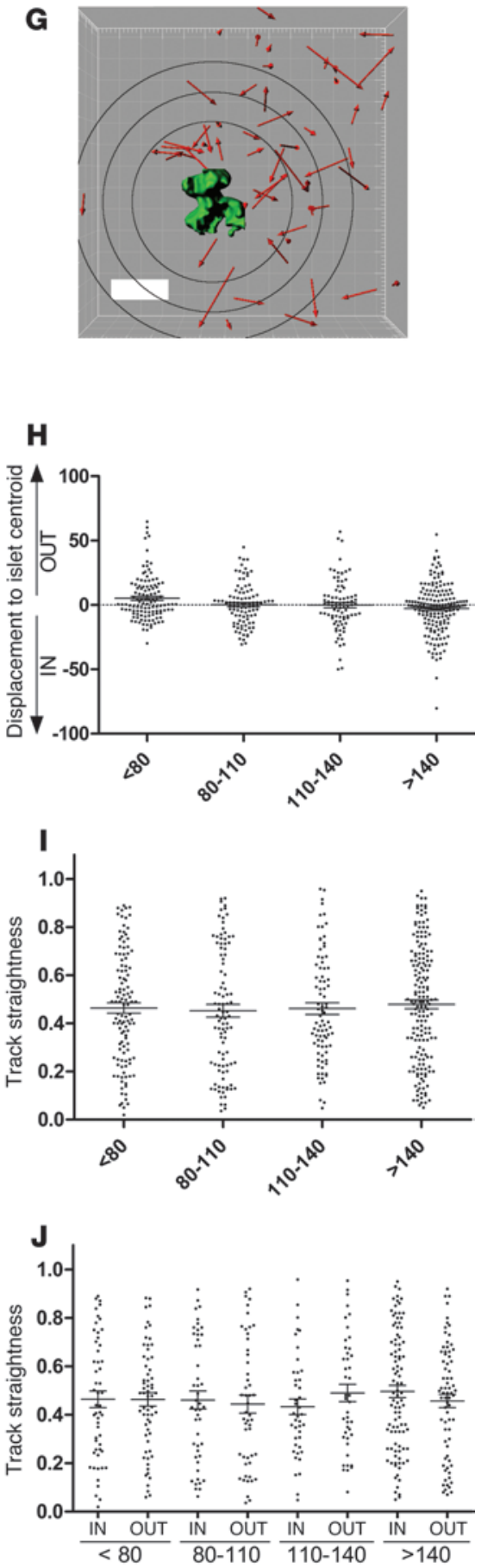

Figure 4

CTLs exhibit random walk motility in the exocrine pancreas. (A-E) Images from time-lapse sequences with tracked CTLs in magenta. Green clusters represent $\beta$ cell mass. Tracks identify past 5 cell positions. Only cells that were tracked in 5 consecutive frames were included for analysis. $\mathbf{C}$ corresponds to Supplemental Video 6 and E with Supplemental Video 7. (F; corresponds to Supplemental Video 8) Analogous setup and analysis but using in vivo peptide/ adjuvant activation (ref. 31). Red lines show linear regression plot. (G) CTL stratification strategy for chemotaxis analysis according to distance from islet centroid. Red arrows represent net CTL displacement, green is isosurfaced islet contour from which centroid position was derived, and black lines represent stratification groups. (H) Net displacement relative to islet centroid, categorized according to initial position from centroid ( $\mu \mathrm{m}$ ). (I) Plot of track straightness across stratified CTL. (J) Comparison of track straightness between CTLs that display net inward versus outward movement relative to the islet centroid. $\mathbf{H}-\mathbf{J}$ represent pooled data from 3 individual time sequences; no significant differences were found using 1 -way ANOVA. Dimensions: A, $w / h=0.91 / d=6 / z=10 ; \mathbf{B}, w / h=1.52 / d=6 / z=24 ; \mathbf{C}, w / h=0.52 / d=6 / z=17 ; \mathbf{D}, w / h=0.80 / d=6 / z=15 ; \mathbf{E}, w / h=0.27 / d=5 / z=24$; $\mathbf{F}, w / h=0.69 / d=6 / z=14 ; t=30$ s for all series. Graphs show means \pm SEM. Scale bars: $50 \mu \mathrm{m}(\mathbf{C}-\mathbf{G}) ; 100 \mu \mathrm{m}(\mathbf{A}$ and $\mathbf{B})$. 

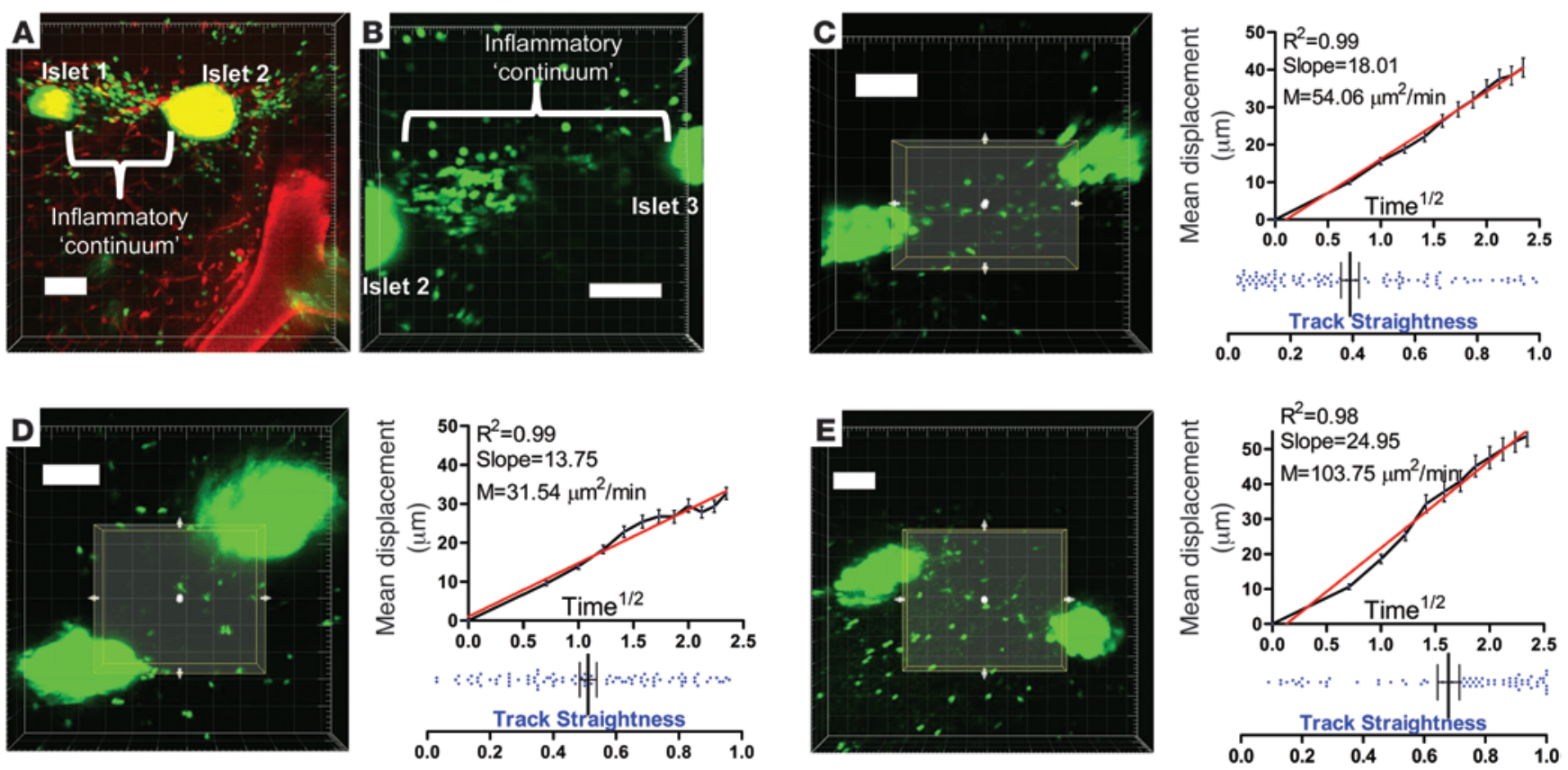

Figure 5

Unimpeded but nondirectional CTL migration between anatomically nearby islets. (A and B) During imaging, CTLs were often found to establish a 3D continuum between adjacent islets. A and $\mathbf{B}$ represent 3 adjacent islets (numbered islet 1, 2, and 3 ) captured in 2 individual Z-stacks over time. B corresponds to Supplemental Video 9. (C-E) 3D regions of interest were drawn in these inter-islet zones, and cellular motility was analyzed within these regions. D corresponds to Supplemental Video 10. Red lines show linear regression plot. Image dimensions: A, $w / h=1.52 / d=6 / z=22 ; \mathbf{B}, w / h=0.70 / d=6 / z=22 ; \mathbf{C}, w / h=1 / d=6 / z=22 ; \mathbf{D}, w / h=1.11 / d=6 / z=16 ; \mathbf{E}, w / h=1.51 / d=5 / z=28$. Horizontal bars are means and associated error bars represent the SEM. Scale bars: $100 \mu \mathrm{m}$.

ited various degrees of CTL motility, no evidence for consistently linear CTL movement was obtained. Overall track straightness was comparable to the values presented in Figure $4(P=0.8325$; pooled data from Figure 4, A-E, versus Figure 5, C-E). Instead, the linear correlation of the displacement versus time ${ }^{1 / 2}$ plots showed that motility could be characterized as "random," analogous to CTLs migrating within the exocrine tissue, as analyzed in Figure 4. We conclude that, while no migratory inhibition is observed that limits CTL trafficking between adjacent islets, no apparent pathways of inter-islet CTL migration exist.

Constrained CTL motility and infrequent formation of stable $\beta$ cell contacts within islets. In order to visualize the kinetic behavior of CTLs within pancreatic islets, an experimental setup was designed that permits spectral separation of fluorescent signal emanating from CTLs, $\beta$ cells, and vasculature. As depicted in Figure 1C, CTLs can be observed to accumulate within and around the $\beta$ cell population. Intra-islet CTL populations from 3 individual prediabetic animals were imaged at cellular resolution (Figure 6, A-C, and corresponding Supplemental Videos 11-13). At digital zoom levels equivalent to those used in Supplemental Videos 12 and 13, a fraction of the infiltrating CTLs were seen to establish long-lasting interactions with the $\beta$ cells, while many more CTLs rapidly migrated through and scanned the islet milieu. For each time series, 5 representative contact regions are included in Figure 6, A-C; these demonstrate the round cellular shape of most CTLs that are contacting $\beta$ cells, which is indicative of their arrest. Figure 6B shows examples of CTL arrest that last for the entire acquisition time of 30 minutes. As an illustration of intense local CTL accumulation, images were recorded showing $\beta$ cells that were almost entirely surrounded by CTLs (Figure 6D).
The results from Figure 1D should hereby be kept in mind in the sense that other leukocyte populations likely block CTL access to the $\beta$ cell surface and may account for the relatively limited frequency of arrest. In the absence of $\beta$ cell antigens, using mIP-GFP single transgenic mice, no such infiltration or arrest within islets was observed (Figure 6E), highlighting the antigen-specific nature of insulitis induction in our model. Only a small number of anti-viral CTLs could be found around days 7-8 after infection, suggesting that CTL retention within target organs critically depends on the availability of antigen and that upon antigen depletion efflux occurs remarkably fast (see also data in full-blown diabetic animals below).

The tracking results of time sequences derived from intra-islet CTL populations quantitatively confirmed the findings outlined above (Figure 6, F-I). Mean track velocities were significantly lower than those associated with acinar CTLs (Figure 6F; $P<0.0001$ for pooled data from Figure 4, A-E, versus Figure 6, A-C), while track straightness was only mildly decreased (Figure 6G; $P=0.0125$ for pooled data from Figure 4, A-E, versus Figure 6, A-C). Intra-islet arrest coefficients were significantly higher than in the exocrine tissue (Figure 6H; $P<0.0001$ for pooled data from Figure 4, A-E, versus Figure $6, \mathrm{~A}-\mathrm{C})$, although the fraction of CTLs that maintained stable arrest for more than half of the imaging time was still relatively minor. Displacement versus time ${ }^{1 / 2}$ plots associated with intra-islet CTLs reproducibly deviated from linearity and exhibited plateaus indicative of constrained motility patterns (Figure 6I). This series of experiments thus shows that CTL migration patterns change from a random, Brownian walk in exocrine regions to a slower, constrained motility accompanied by increased yet infrequent cellular arrest within antigen-sufficient $\beta$ cell zones. 

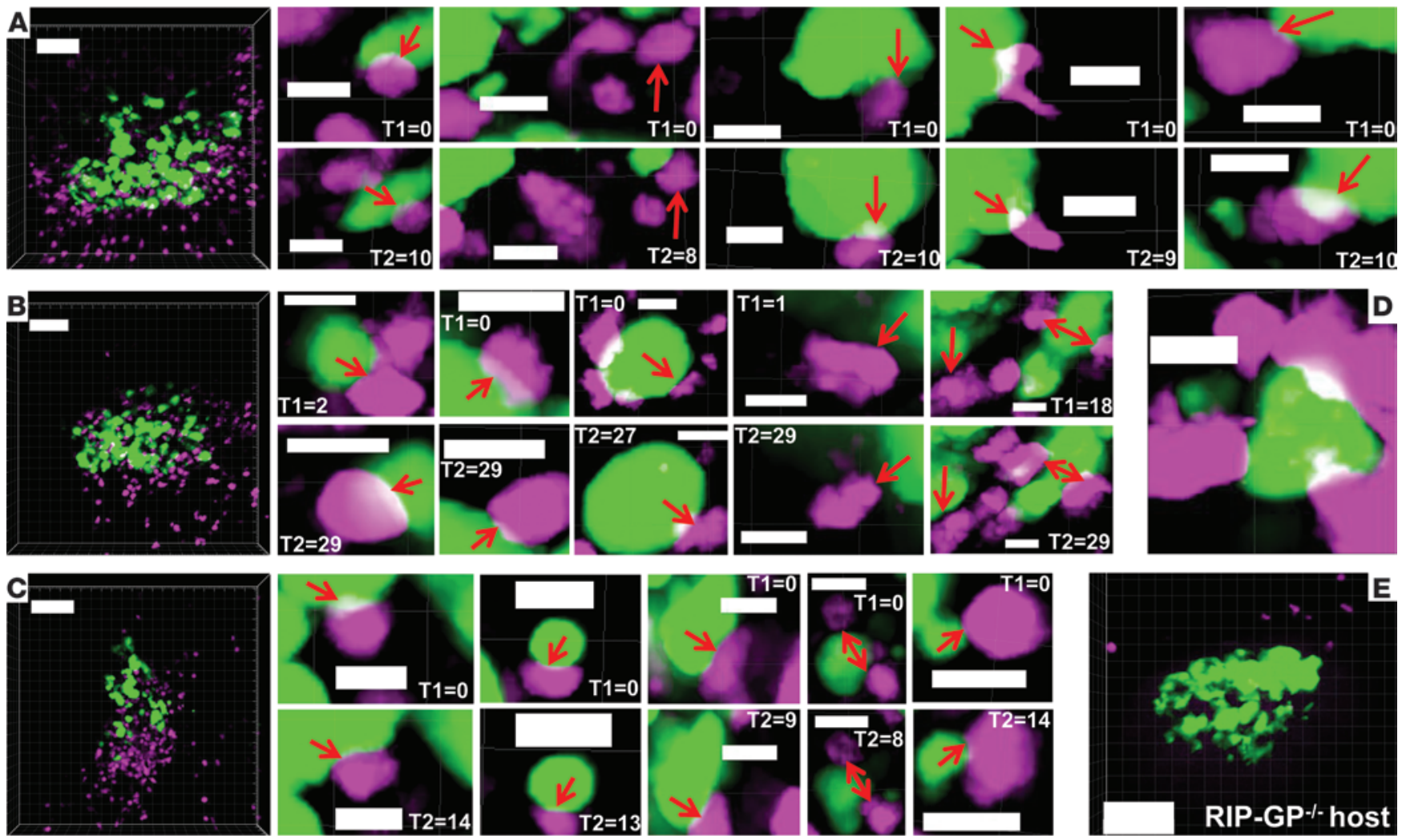

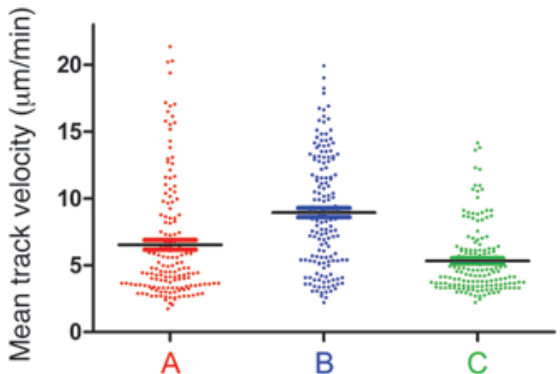

H

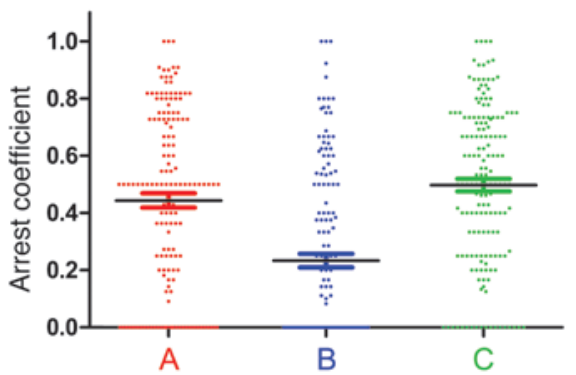

G

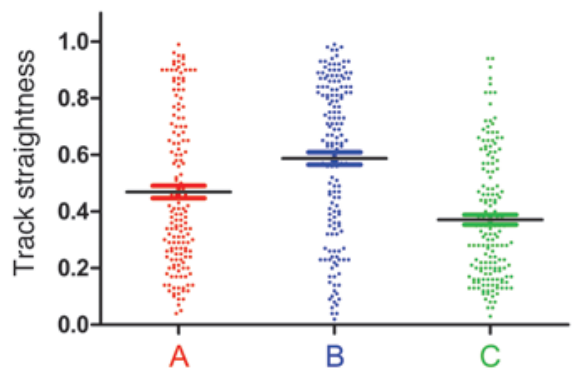

I

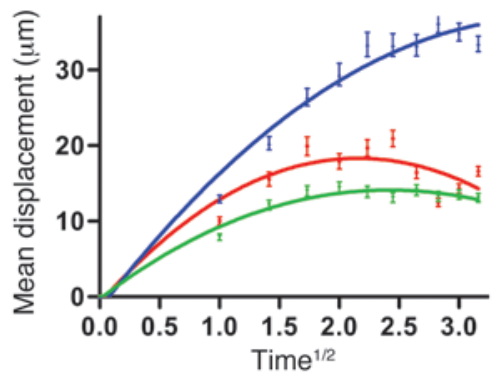

Figure 6

CTL motility within pancreatic islets imaged at cellular resolution. (A-C) Pancreatic islet (green, GFP) and infiltrating CTL (magenta, CFP) populations from 3 individual mice were imaged at the highest digital magnification that would fully encompass $\beta$ cell mass and associated CTLs. Dimensions: $\mathbf{A}, w / h=0.79 / d=5 / z=22 ; \mathbf{B}, w / h=0.88 / d=5 / z=20 ; \mathbf{C}, w / h=0.91 / d=5 / z=25$. Acquisition times are 10 minutes, 29 minutes, and 14 minutes, respectively, with $t=60 \mathrm{~s}$. A corresponds to Supplemental Video 11. Five representative, high-resolution examples are included for each series that document CTL- $\beta$ cell interactions (first frame [T1 in min] and last frame of contact [T2 in min]). Supplemental Videos 12 and 13 correspond to magnified zones from $\mathbf{A}$ and $\mathbf{B}$, respectively. (D) Enlarged region from $\mathbf{C}$ showing a $\beta$ cell that is almost entirely engulfed by $\mathbf{C T L s}$. (E) Control experiment using mice with fluorescently labeled but antigen-deficient $\beta$ cells. A small exocrine population of CTLs can be observed. One frame is shown from a 14-minute sequence during which none of the CTLs shown here infiltrated the islet (representative of duplicate control experiments). Dimensions: $w / h=0.75 / d=5 / z=24$. (F-I) Kinetic CTL population parameters obtained via 3D cellular-tracking analysis in Imaris. Color coding correlates with the image sequences in A-C. Curves in I were generated via nonlinear regression analysis in GraphPad and, in combination with $R^{2}$ values, reveal deviation from linearity and thus constrained motility of the intra-islet CTL population. Horizontal bars are means and associated error bars represent the SEM. Scale bars: $80 \mu \mathrm{m}$ (A-C and E); $10 \mu \mathrm{m}$ (D). 
Slow induction of $\beta$ cell death and rapid contraction of the CTL response upon antigen depletion. The clinical consequence of $\beta$ cell destruction, hyperglycemia, is thought to occur when a critical amount of $\beta$ cell mass has been destroyed by the immune process. Given our findings on constrained CTL motility and arrest, we next investigated the temporal rate of $\beta$ cell death occurring in these islets. In our previous studies in a peptide/adjuvant-induced variant of the RIP-LCMV. GP model, we failed to notice events of $\beta$ cell death despite the acute onset of hyperglycemia (31). Speier et al. previously showed that transgenic MIP-GFP $\beta$ cells transplanted in the anterior eye chamber lose their GFP content within 24 hours after alloxan treatment (30), and GFP content has been shown to be useful for real-time detection of cellular death (42). We therefore reasoned that loss of GFP should serve as a valid readout in our studies.

Loss of $\beta$ cell-related GFP signal was initially recorded on 3 occasions in different MIP-GFP/RIP-GP animals supplemented with P14.GFP CTLs (Figure 7, A-C, and corresponding Supplemental Video 14). Islets from naive control animals were monitored for 40 minutes at maximum laser intensity to exclude confounding effects of laser toxicity or surgery (Figure 7D). The latter confirmatory experiment aligns with our previous data, in which islets were imaged for durations of over 4 hours in the absence of GFP decay (31). CTL tracking demonstrated the presence of large numbers of unique tracks within the surrounding acinar tissue $(19,17$, and 6 $\mathrm{CTL} / \mu \mathrm{m}^{3}$ imaging volumes for $\mathrm{A}, \mathrm{B}$, and $\mathrm{C}$ sequences, respectively). This number is by all means an underestimation of the actual CTL number, as it excludes islet-infiltrating CTLs and unlabeled leukocytes. Taking into account the known fate of the $\beta$ cell mass at the time of imaging (mice turn diabetic the next day at the latest), this suggests that CTL killing on a cellular basis is associated with a temporal course on the order of hours and may in fact constitute the limiting factor as suggested by modeling reports (23). These data therefore confirm in silico data as well as empirical results by Breart and coworkers indicating the generally protracted time course of CTL killing in peripheral target tissues $(23,26)$.

Next, we investigated whether stable interactions between CTL and $\beta$ cells, as observed in Figure 6 , are strictly associated with $\beta$ cell death and loss of GFP signal. To achieve spectral separation of CTLs and $\beta$ cells, the CTLs were labeled with CFP in these experiments. In over 12 hours of image acquisition using 12 individual CTL recipients, a total of 8 events of $\beta$ cell death were recorded (data not shown). We found that while some events of GFP loss were associated with CTL contact, other $\beta$ cells underwent decay in the absence of any CTL association (Figure 7E and Supplemental Videos 15 and 16). These observations indirectly confirm results from conventional studies showing that $\beta$ cell death occurs as a consequence of the combined action of contact-dependent cytotoxicity and contactindependent mechanisms, such as cytokine cytotoxicity.

Finally, we asked how the depletion of antigen and corresponding onset of hyperglycemia translated to the kinetic behavior of CTLs in the pancreas. In mice that were in the first day of full-blown clinical hyperglycemia (blood glucose $>600 \mathrm{mg} / \mathrm{dl}$; generally corresponds to images depicted in Figure 1D), 2 patterns were observed. In mice in which significant CTL numbers were still observed $\left(5 \mathrm{CTL} / \mu^{3} \mathrm{~m}^{3}\right.$ imaging volume; Figure $\left.7 \mathrm{~F}\right)$ in the presence of remnant $\beta$ cells, tracking analysis showed that this population exhibited random motility, as recorded in exocrine populations included in Figure 4 (Figure 7, H and I). Moreover, Supplemental Video 17 shows that CTLs do not arrest at or are confined to the sole remaining $\beta$ cell within the imaging volume. These findings sug- gest that a minor population of $\beta$ cells survives the immune-mediated attack, possibly due to restoration of regulatory mechanisms or CTL exhaustion, and confirm the notion that isolated clusters of insulin-sufficient cells can often be observed histologically even in long-standing diabetic mice $(11,43)$ and T1D patients (44).

The alternative and dominant pattern in the full-blown diabetic pancreas, and the only pattern observed after day 9 after infection, was the presence of a limited CTL population $\left(0.4 \mathrm{CTL} / \mu^{3} \mathrm{imag}^{2}\right.$ ing volume; Figure $7 \mathrm{G}$ and corresponding Supplemental Video 18) with markedly different motility features (Figure 7, $\mathrm{H}$ and I). While this minor population also displayed a random walk, mean velocity, track straightness, and arrest coefficient differed significantly.

These findings suggest that CTLs within antigen-depleted pancreas tissue from diabetic animals briefly adopt a random walk pattern shortly after clinical onset, but rapidly undergo either death or egress. The minor remaining population is found to slowly dwell within the tissue while lacking any directionality.

\section{Discussion}

The data presented here describe the kinetic behavior of an antiviral CTL population that redirects its cytotoxicity toward $\beta$ cells and becomes diabetogenic after viral clearance. Pathophysiologic mechanisms that are thought to be relevant to T1D, such as genetic predisposition leading to defective thymic selection and escape of low-affinity TCR clones into the periphery, are obviously not at play in the model employed here. The aim of our current study was therefore not to unravel mechanisms of initial immune synapse formation or priming of diabetogenic CTL clones. Indeed, it is still unknown whether initial antigen encounter occurs within pancreatic draining lymph nodes, directly within islets, or even in unrelated anatomic sites such as the gut (45). We do know for certain that inflamed islets from recent-onset T1D patients contain a significant diabetogenic CTL compartment $(1,4)$. Therefore, we focused our efforts on tracking the kinetic behavior of diabetogenic CTLs after activation and expansion, starting from extravasation into the target tissue to the point of $\beta$ cell antigen depletion and onset of clinical hyperglycemia. Similar to a recent study by Kang et al., who used the P14 TCR-transgenic line as a traceable representative to track antiviral CTL responses (28), we used these cells to trace the diabetogenic CTL response after viral clearance.

Observation of the relatively short-lived initial interactions of leukocytes with vascular endothelium requires image acquisition at higher frame rates and resolution than achievable with our intravital 2-photon microscopy setup (e.g., spinning-disk confocal microscopy, as used in ref. 46). The arrested CTLs in the present study thus likely represent the subsequent step of endothelial transmigration, i.e., firm adhesion to the vessel wall, which is a rate-limiting step controlling leukocyte recruitment in inflammation (47). Following firm attachment to the endothelial surface, lymphocytes must crawl through interendothelial junctions and cross the underlying basement membrane in order to successfully enter tissue, a process referred to as diapedesis, transmigration, or extravasation (34). This process is generally accompanied by increased vascular permeability, a phenomenon that has been described in other models for autoimmune inflammation such as EAE (48). The blood flow rate in postcapillary venules is known to be considerably lower than in arterioles, and this mechanical property conveys more suitable grounds for maintenance of weaker initial tethering contacts, thus reducing the likelihood that the shear force of the flowing blood will dislodge the tethered lymphocyte. The alternative explanation for our find- 

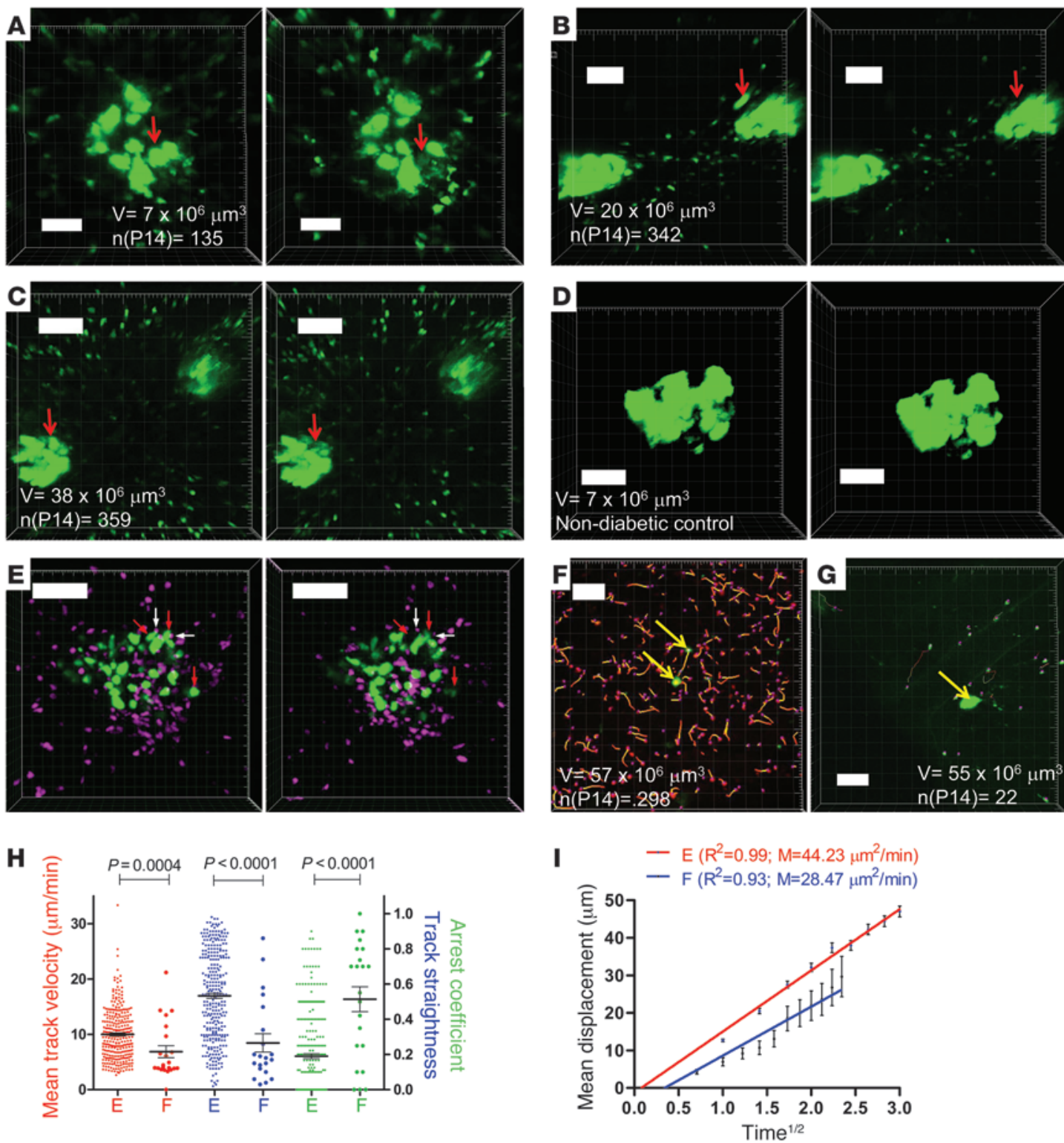

Figure 7

$\beta$ cell death and CTL motility after antigen depletion. (A-C) Events of $\beta$ cell death with loss of GFP as a readout measure (red arrows). A corresponds to Supplemental Video 14. B is derived from the same sequence used in Figure $5 \mathrm{C}$. Imaging volume $\mathrm{V}$ and number of unique $\mathrm{CTL}$ tracks are indicated. Dimensions: $\mathbf{A}, w / h=0.58 / d=6 / z=21 ; \mathbf{B}, w / h=1 / d=6 / z=22 ; \mathbf{C}, w / h=1.08 / d=6 / z=10 ; t=30 \mathrm{~s}$ for all sequences. (D) Representative control islet from naive animal imaged at maximum laser intensity; $w / h=0.51 / d=5 / z=22$. (E) Association of arrested CTL (CFP, pseudocolored magenta; white arrows) with dying $\beta$ cells (GFP, green; red arrows). E corresponds to Supplemental Videos 15 and 16 ; $w / h=0.86 / d=5 / z=22$. $(F-I)$ Representative images from full-blown diabetic mice and corresponding tracking results. Imaging volume $V$ and number of unique CTL tracks are indicated. Supplemental Video 17 shows an enlarged region of $\mathbf{F}$, while $\mathbf{G}$ corresponds to Supplemental Video 18 , which includes vascular staining. Representative of 3 individual experiments. Dimensions: $\mathbf{F}, w / h=1.52 / d=5 / z=20 ; \mathbf{G}, w / h=1.52 / d=5 / z$ $=18$. Yellow arrows in $\mathbf{F}$ and $\mathbf{G}$ indicate remaining $\beta$ cells. $P$ values were obtained with Mann-Whitney analysis. Horizontal bars are means and associated error bars represent the SEM. Scale bars: $100 \mu \mathrm{m}(\mathbf{B}, \mathbf{C}, \mathbf{E}-\mathbf{G}) ; 50 \mu \mathrm{m}$ (A and D).

ings is that the arrested CTLs are engaged in TCR-induced immune synapse formation and are in fact actively exerting cytotoxic action against the endothelial cells. Savinov and coworkers showed that endothelial cells in the vicinity of islets actively present islet antigens to $\mathrm{CD}^{+} \mathrm{T}$ cells and are consequentially targeted, potentially leading to profound vascular leakage (36). In the acute, virus-induced RIPGP model employed here, we did not find evidence for this phenom- enon, as extensive TUNEL staining showed that apoptosis is limited to the islet cells and associated infiltrate rather than endothelial cells. We therefore interpret the observed CTL arrest in the postcapillary venules as the firm adhesion step preceding transendothelial migration. Since extravasation constitutes a decisive, irreversible step in autoimmune infiltration, therapeutic strategies aimed at this junction have been proposed (49). Our present data further suggest 
that the induction of vascular permeability through environmental factors such as viral infection may be a crucial accelerator in T1D progression. Indeed, ongoing islet autoimmunity appears to rapidly culminate in hyperglycemia in both animal models and T1D patients following enteroviral infection $(8,50)$.

Once the diabetogenic CTLs have entered the pancreas, we find that they are able to efficiently traverse the exocrine tissue by random walk. It has been proposed that pancreatic islets in T1D may become a rich source of chemoattractants upon viral infection, which in turn serve by directing the diabetogenic CTLs to the $\beta$ cell population (51). Moreover, viral agents have been hypothesized as a potential cause for islet-specific hyperexpression of chemokines in human patients $(52,53)$. Based on this hypothesis, one would thus expect to observe some degree of CTL directionality, especially in the proximity of islets. We find that active, directional CTL recruitment is not discernible and suggest that CTLs may migrate through and scan the exocrine tissue until they reach the pancreatic islets by chance. What evidence do we currently have to assume that viral infections infect pancreatic islets and induce chemokine gradients specifically toward them rather than inducing chemokine patterns across the entire organ? Enterovirus infection has only occasionally been found in islets from T1D patients (5). Much like enteroviruses, which are considered prime etiologic candidates in T1D, LCMV is not known to preferentially infect pancreatic islets, but rather has a tropism for the entire organ. Based on these considerations and our finding that CTL motility remains unaltered when a noninfectious activation protocol is implemented, the scenario of islet-specific establishment of chemokine attraction seems implausible. It should be noted, however, that limitations of our approach in terms of imaging volume and duration will make chemotactic effects that act over longer distances and times go unnoticed. Mechanisms that are not islet specific, such as the induction of vascular leakiness and enhanced CTL recruitment to the pancreas, may rather account for diabetes acceleration that is achieved after enteroviral infection of prediabetic NOD mice (50). It is also tempting to speculate that the focal, lobular pattern of insulitis progression in T1D may in part depend on 2 of the phenomena described here: proximity of islets to the "leaky" sites of CTL extravasation and local differences in CTL motility that lead to various probabilities of antigen encounter.

An important consequence of the unimpeded motility of CTLs in pancreas tissue may be that aberrant extravasation exposes multiple pancreatic islets to their cytotoxic action. Whereas we did not detect directional CTL migration between adjacent islets, CTLs within these inter-islet zones were not found to be physiologically impeded from trafficking between such sites. However random CTL motility in the acinar islet periphery may be, once CTLs enter the islet milieu, their kinetic behavior drastically changes. Our experiments showed profound migratory constraint, and analysis of motility parameters showed that CTLs became "trapped" within the direct vicinity of the $\beta$ cells. We noted a relative infrequency of stable arrest and contact formation with $\beta$ cells. Several explanations may account for this observation, one of which might be restricted access to antigenic sites owing to dense accumulation of many types of leukocyte populations, as presented in Figure 1D. These findings mirror in silico data predicting that decreases in mean CTL velocity and motility only occur in the presence of CTLtarget cell interactions (23). Once $\beta$ cell antigen is depleted, CTLs rapidly adopt random walk patterns and subsequently disappear from the tissue within hours, once again highlighting the antigendependent nature of their retention in the target tissue.
Finally, our findings bear significance to the understanding of the temporal features of CTL effector function. Flow cytometrybased "in vivo" cytotoxicity assays such as employed by Barber et al. offer a valuable estimation of CTL killing efficacy, but are potentially flawed by the delay between cell isolation and flow cytometry analysis (54). Ideally, analysis of CTL efficacy requires models that capture the anatomical complexity of living target tissue and should go beyond analysis within secondary lymphoid tissues. Only a handful of advanced imaging studies have addressed the efficacy of CTL killing in vivo. Of particular note is the study by Breart and coworkers, who estimated the time required for CTL killing of a tumor cell in their model at approximately 6 hours (26). We argue based on our data that a similar time range may apply for CTL-mediated $\beta$ cell destruction. Detailed in silico CTL modeling studies revealed that killing follows a linear relationship with target cell frequency and saturates with CTL levels and that the duration of the actual killing event is the rate-limiting step. (23). Concretely, it was suggested that during a simulated time period of 300 minutes. a CTL would kill on average 2-7 target cells, depending on the velocity and killing duration. The instances of $\beta$ cell death that were recorded in our work here, in conjunction with the prolonged duration of CTL arrest at the $\beta$ cell junction, support a scenario of slow and rate-limiting killing. It should be noted, however, that the net involvement of contact-dependent toxicity may be underestimated in this setup. Loss of GFP can be assumed to be the final step in an apoptotic cascade and may occur independently of CTL contact in $\beta$ cells that have reached the final stage of the apoptotic cascade. This does not exclude that these $\beta$ cells were subjected to contact-dependent CTL cytotoxicity earlier, which may partially explain the abundance of arrested CTLs as presented in Figure 6. An alternative and equally plausible explanation is that a fraction of the arrested CTLs are actually in contact with unlabeled cells, such as dendritic cells. Given the vast numbers of CTLs responsible for diabetes onset in the acute RIP-LCMV.GP model and the astoundingly low frequency thereof in prediabetic, autoantibody-positive individuals, this may at least partially explain the long prediabetic phase that typically precedes clinical T1D onset (55).

In conclusion, we show here that diabetogenic CTLs display unimpeded motility within exocrine tissue upon extravasation, adopt a constrained motility pattern only within pancreatic islets, and induce $\beta$ cell death at a relatively slow cellular rate. We propose that viral agents may act by facilitating diabetogenic CTL extravasation into the pancreas rather than orchestrating directed attraction specifically toward islets. Therapeutic strategies aimed at preventing CTL extravasation in susceptible or recent-onset individuals may therefore be advisable.

\section{Methods}

Mice. We crossed B6 mice ubiquitously expressing GFP (C57BL/6-Tg(ACTBEGFP)1Osb/J; ref. 56) with P14 mice (32), which express a TCR specific for the GP33 CD8 ${ }^{+}$epitope of the LCMV. Rag2 $2^{--}$actin-CFP/P14 mice were a gift of Ellen Robey (University of California, Berkeley, Berkeley, California, USA) and were previously described (57). Mice expressing GP antigen from the LCMV-WE (13) strain under control of the rat insulin promoter (RIP-GP) were bred to a strain with $\beta$ cell-restricted GFP expression (MIP-GFP) (33).

Diabetes induction protocol. Single-cell suspensions were prepared from spleen. Red blood cell lysis was performed, and untouched $\mathrm{CD}^{+} \mathrm{T}$ cells were isolated via negative depletion with Dynal magnetic beads in conjunction with antibodies against B220, CD16/32, CD4, and MHC II. All antibodies used were purchased from BD. Purity was confirmed by flow cytometry and 
was consistently in the range of $95 \%$ of total $\mathrm{TCR}^{+} \mathrm{CD}^{+}$cells. On day 0 , a single-cell suspension of $1 \times 10^{6}$ purified P14 splenocytes was transferred into recipient mice via retroorbital injection. Viral infection with LCMV strain Armstrong was performed on day 1. For peptide/adjuvant induction, on days 1 and 3, mice were i.p. injected with $2 \mathrm{mg}$ of GP33 peptide (NH2KAVYNFATM-COOH; Abgent) in ultrapure water containing $50 \mu \mathrm{g}$ of $\mathrm{CpG}$ adjuvant (Sigma-Aldrich). Finally, on day 6 , mice received $500 \mu \mathrm{g}$ of polyinosinic/polycytidylic acid (poly I:C) i.p. (Sigma-Aldrich). All imaging was done on days 7 and 8 (prediabetic) or day 9 (diabetic), as indicated. Blood glucose values were measured prior to imaging using OneTouch meters and strips.

Immunofluorescent staining. Tissues were embedded and snap-frozen in OCT compound, and 6- $\mu \mathrm{m}$ sections were cut with a cryostat. Sections were fixed and stained with unlabeled primary antibodies (BD) and detected with Alexa Fluor 488-labeled, highly cross-adsorbed secondary antibodies against the host species of the primary antibody (Invitrogen). The in situ cell death detection kit from Roche was used for TUNEL assay and used as per the manufacturer's instructions. Sections were mounted with ProLong Antifade medium with DAPI, and images were acquired using a Nikon Eclipse 80i fluorescence microscope equipped with a Plan Apo ×20, N.A. 0.75 air objective.

Surgery and temperature control. A detailed procedure was published previously and is publicly available (31). Briefly, mice were anesthetized and splenectomy was performed. The pancreatic tail region was exposed on a custommade imaging device. The imaging reservoir's temperature was controlled by a recirculation system based on a Gaymar $\mathrm{T} / \mathrm{Pump}$. Local temperature at the tissue was checked with a sensitive probe (Warner Instruments).

2-Photon imaging and analysis. We used a Leica SP5 microscope system equipped with an IR Laser Chameleon Ultra ps, tunable from 690 to 1040 $\mathrm{nm}$ and used here at $900 \mathrm{~nm}$. For this study, we used 3 channels with nondescanned detectors with filter sets suitable for separation of GFP $(513 / 17 \mathrm{~nm})$, CFP $(475 / 20 \mathrm{~nm})$, and Texas Red $(692 / 40 \mathrm{~nm})$ in combination with 506 (CFP/GFP) and $560 \mathrm{~nm}$ (GFP/Texas Red) dichroic mirrors. We used an Olympus Super 20 (XLUMPLFL20XW, NA0.95) water dipping objective controlled by a Piezo $Z$ focus element (Piezosystem Jena). All image sequences used for tracking analysis used z-stepping of $6 \mu \mathrm{m}$ or less and time resolutions of 30 seconds or 1 minute. as indicated in the legends. Postacquisition enhancement (i.e., "normalization" or altering the range of pixel intensity values for viewing purposes), maximum intensity projection (MIP), and panel combination were performed using Imaris software (Bitplane) or ImageJ. The Turboreg and Stackreg plugins (58) were used for registration within Z-stacks (i.e., elimination of breathing artifact). 3D tracking and isosurfacing was performed using Imaris after correction for tissue drift. True cellular contact was confirmed by looking at the individual Z-planes. In some videos, spectral overlap at the islet site was corrected for by subtracting the "red" signal from the "green" channel, rendering all islets uniformly green for the sake of clarity. Finally, image sequences were converted to TIFF or AVI format. Image dimensions are provided in microns for each image and video in the legends and defined as follows: $w$, pixel width; $h$, pixel height; $d$, voxel depth or z-stepping; $z$, number of z-planes captured; and $t$, time resolution.

The confinement ratio corresponds to the ratio of the distance between the initial and the final positions of each cell to the total distance covered by the same cell. The arrest coefficient is defined as the proportion of time each cell's instantaneous velocity (calculated for every time interval) is less than $3 \mu \mathrm{m} / \mathrm{min}$. For analysis of chemotaxis, islets were isosurfaced and centroid position was determined. Euclidean distances between starting and ending positions from CTL-associated tracks were calculated in Excel, and net displacement versus centroid was calculated. Visualization of vasculature was achieved by the i.v. injection of $250 \mu \mathrm{g}$ of $70 \mathrm{kDa}$ Texas Red-labeled dextran in PBS (Invitrogen).

Statistics. Regression analysis, calculation of $R^{2}$, Mann-Whitney, and 1-way ANOVA tests were performed using GraphPad Prism software. $P<0.05$ was considered significant.

Study approval. All animal procedures were approved by the Animal Care Committee of the La Jolla Institute for Allergy and Immunology (protocol nr. AP121-MvH3-0510).

\section{Acknowledgments}

We thank Malina McClure for maintenance of our mouse colonies and Priscilla Colby for administrative assistance. We are grateful to William B. Kiosses (The Scripps Research Institute) for help with image analysis. This work was funded by Juvenile Diabetes Research Foundation Scholar Award 16-2007-370 to M. von Herrath and supported by the Brehm Coalition.

Received for publication June 1, 2011, and accepted in revised form October 19, 2011.

Address correspondence to: Matthias von Herrath, 9420 Athena Circle, La Jolla, California 92037, USA. Phone: 858.752.6892; Fax: 858.752.6993; E-mail: matthias@liai.org.

Ken T. Coppieters's present address is: Unit for Molecular Immunology and Inflammation, Ghent University Hospital, Ghent, Belgium.
1. Coppieters KT, von Herrath MG. Histopathology of type 1 diabetes: old paradigms and new insights. Rev Diabet Stud. 2009;6(2):85-96

2. Foulis AK, Farquharson MA, Meager A. Immunoreactive alpha-interferon in insulin-secreting beta cells in type 1 diabetes mellitus. Lancet. 1987;2(8573):1423-1427.

3. Dotta F, et al. Coxsackie B4 virus infection of beta cells and natural killer cell insulitis in recent-onset type 1 diabetic patients. Proc Natl Acad Sci U S A. 2007;104(12):5115-5120.

4. Skowera A, et al. CTLs are targeted to kill beta cells in patients with type 1 diabetes through recognition of a glucose-regulated preproinsulin epitope. J Clin Invest. 2008;118(10):3390-3402.

5. Richardson SJ, Willcox A, Bone AJ, Foulis AK, Morgan NG. The prevalence of enteroviral capsid protein vp1 immunostaining in pancreatic islets in human type 1 diabetes. Diabetologia. 2009;52(6):1143-1151.

6. Clements GB, Galbraith DN, Taylor KW. Coxsackie $B$ virus infection and onset of childhood diabetes. Lancet. 1995;346(8969):221-223.

7. Oikarinen S, et al. Enterovirus RNA in blood is linked to the development of type 1 diabetes. Diabetes. 2011;60(1):276-279.

8. Stene LC, et al. Enterovirus infection and progression from islet autoimmunity to type 1 diabetes: the Diabetes and Autoimmunity Study in the Young (DAISY). Diabetes. 2010;59(12):3174-3180.

9. Oikarinen S, et al. Enterovirus RNA in blood is linked to the development of type 1 diabetes. Diabetes. 2011;60(1):276-279.

10. Yeung WC, Rawlinson WD, Craig ME. Enterovirus infection and type 1 diabetes mellitus: systematic review and meta-analysis of observational molecular studies. BMJ. 2011;342:d35.

11. von Herrath MG, Dockter J, Oldstone MB. How virus induces a rapid or slow onset insulin-dependent diabetes mellitus in a transgenic model. Immunity. 1994;1(3):231-242.

12. Oldstone MB, Nerenberg M, Southern P, Price J, Lewicki H. Virus infection triggers insulin-dependent diabetes mellitus in a transgenic model: role of anti-self (virus) immune response. Cell. 1991;65(2):319-331.

13. Ohashi PS, et al. Ablation of "tolerance" and induction of diabetes by virus infection in viral antigen transgenic mice. Cell. 1991;65(2):305-317.

14. Ohashi PS, et al. Induction of diabetes is influenced by the infectious virus and local expression of MHC class I and tumor necrosis factor-alpha. JImmunol. 1993;150(11):5185-5194.

15. Holz A, Dyrberg T, Hagopian W, Homann D, von Herrath M, Oldstone MB. Neither B lymphocytes nor antibodies directed against self antigens of the islets of Langerhans are required for development of virus-induced autoimmune diabetes. J Immunol. 2000;165(10):5945-5953.

16. Martin S, et al. Development of type 1 diabetes despite severe hereditary B-lymphocyte deficiency. N Engl J Med. 2001;345(14):1036-1040.

17. Christen U, McGavern DB, Luster AD, von Herrath $\mathrm{MG}$, Oldstone $\mathrm{MB}$. Among CXCR3 chemokines, IFN-gamma-inducible protein of 10 $\mathrm{kDa}$ (CXC chemokine ligand (CXCL) 10) but not monokine induced by IFN-gamma (CXCL9) imprints a pattern for the subsequent development of autoimmune disease. J Immunol. 2003; 171(12):6838-6845.

18. Roep BO, et al. Islet inflammation and CXCL10 in recent-onset type 1 diabetes. Clin Exp Immunol. 
2010;159(3):338-343

19. Mempel TR, Henrickson SE, Von Andrian UH. T-cell priming by dendritic cells in lymph nodes occurs in three distinct phases. Nature. 2004;427(6970):154-159.

20. Henrickson SE, et al. T cell sensing of antigen dose governs interactive behavior with dendritic cells and sets a threshold for $\mathrm{T}$ cell activation. Nat Immunol. 2008;9(3):282-291.

21. Beuneu $\mathrm{H}$, et al. Visualizing the functional diversification of CD8+ $\mathrm{T}$ cell responses in lymph nodes. Immunity. 2010;33(3):412-423.

22. Bousso P, Robey E. Dynamics of CD8+ T cell priming by dendritic cells in intact lymph nodes. Nat Immunol. 2003;4(6):579-585.

23. Graw F, Regoes RR. Investigating CTL mediated killing with a 3D cellular automaton. PLoS Comput Biol. 2009;5(8):e1000466.

24. Deguine J, Breart B, Lemaitre F, Di Santo JP, Bousso $P$. Intravital imaging reveals distinct dynamics for natural killer and CD8(+) T cells during tumor regression. Immunity. 2010;33(4):632-644.

25. Boissonnas A, Fetler L, Zeelenberg IS, Hugues S, Amigorena S. In vivo imaging of cytotoxic T cell infiltration and elimination of a solid tumor. J Exp Med. 2007;204(2):345-356.

26. Breart B, Lemaitre F, Celli S, Bousso P. Two-photon imaging of intratumoral CD8+ T cell cytotoxic activity during adoptive $\mathrm{T}$ cell therapy in mice. J Clin Invest. 2008;118(4):1390-1397.

27. Kim JV, Kang SS, Dustin ML, McGavern DB. Myelomonocytic cell recruitment causes fatal CNS vascular injury during acute viral meningitis. Nature. 2009;457(7226):191-195.

28. Kang SS, et al. Migration of cytotoxic lymphocytes in cell cycle permits local MHC I-dependent control of division at sites of viral infection. J Exp Med. 2011;208(4):747-759.

29. Coppieters K, von Herrath M. Taking a closer look at the pancreas. Diabetologia. 2008;51(12):2145-2147.

30. Speier $S$, et al. Noninvasive in vivo imaging of pancreatic islet cell biology. Nat Med. 2008;14(5):574-578.

31. Coppieters K, Martinic MM, Kiosses WB, Amirian $\mathrm{N}$, von Herrath $\mathrm{M}$. A novel technique for the in vivo imaging of autoimmune diabetes development in the pancreas by two-photon microscopy. PLoS One. 2010;5(12):e15732.

32. Pircher H, Burki K, Lang R, Hengartner H, Zinkernagel RM. Tolerance induction in double specific
T-cell receptor transgenic mice varies with antigen. Nature. 1989;342(6249):559-561.

33. Hara $M$, et al. Transgenic mice with green fluorescent protein-labeled pancreatic beta cells. $A m \mathrm{~J}$ Physiol Endocrinol Metab. 2003;284(1):E177-E183.

34. Engelhardt B, Wolburg H. Mini-review: Transendothelial migration of leukocytes: through the front door or around the side of the house? Eur J Immunol. 2004;34(11):2955-2963.

35. Ley K, Laudanna C, Cybulsky MI, Nourshargh S. Getting to the site of inflammation: the leukocyte adhesion cascade updated. Nat Rev Immunol. 2007;7(9):678-689.

36. Savinov AY, Wong FS, Stonebraker AC, Chervonsky AV. Presentation of antigen by endothelial cells and chemoattraction are required for homing of insulin-specific CD8+ T cells. J Exp Med. 2003;197(5):643-656.

37. Miller MJ, Wei SH, Cahalan MD, Parker I. Autonomous $\mathrm{T}$ cell trafficking examined in vivo with intravital two-photon microscopy. Proc Natl Acad SciUS A. 2003;100(5):2604-2609.

38. Shakhar G, et al. Stable T cell-dendritic cell interactions precede the development of both tolerance and immunity in vivo. Nat Immunol. 2005;6(7):707-714.

39. Cahalan MD, Parker I. Choreography of cell motility and interaction dynamics imaged by two-photon microscopy in lymphoid organs. Annu Rev Immunol. 2008;26:585-626.

40. Frigerio $S$, et al. Beta cells are responsible for CXCR3-mediated T-cell infiltration in insulitis. Nat Med. 2002;8(12):1414-1420.

41. Okada T, et al. Antigen-engaged B cells undergo chemotaxis toward the $\mathrm{T}$ zone and form motile conjugates with helper T cells. PLoS Biol. 2005;3(6):e150.

42. Strebel A, Harr T, Bachmann F, Wernli M, Erb P. Green fluorescent protein as a novel tool to measure apoptosis and necrosis. Cytometry. 2001;43(2):126-133.

43. von Herrath MG, Oldstone MB. Interferon-gamma is essential for destruction of beta cells and development of insulin-dependent diabetes mellitus. J Exp Med. 1997;185(3):531-539.

44. Keenan HA, et al. Residual insulin production and pancreatic ss-cell turnover after 50 years of diabetes: Joslin Medalist Study. Diabetes. 2010; 59(11):2846-2853

45. van Belle TL, Coppieters KT, von Herrath MG. Type 1 diabetes: etiology, immunology, and therapeutic strategies. Physiol Rev. 2011;91(1):79-118.

46. Nyman LR, et al. Real-time, multidimensional in vivo imaging used to investigate blood flow in mouse pancreatic islets. J Clin Invest. 2008; 118(11):3790-3797.

47. Jung U, Norman KE, Scharffetter-Kochanek K, Beaudet AL, Ley K. Transit time of leukocytes rolling through venules controls cytokine-induced inflammatory cell recruitment in vivo. J Clin Invest. 1998;102(8):1526-1533.

48. Graesser D, et al. Altered vascular permeability and early onset of experimental autoimmune encephalomyelitis in PECAM-1-deficient mice. J Clin Invest. 2002;109(3):383-392.

49. Savinov AY, Burn P. Interference with islet-specific homing of autoreactive $T$ cells: an emerging therapeutic strategy for type 1 diabetes. Drug Discov Today. 2010;15(13-14):531-539.

50. Serreze DV, Ottendorfer EW, Ellis TM, Gauntt CJ, Atkinson MA. Acceleration of type 1 diabetes by a coxsackievirus infection requires a preexisting critical mass of autoreactive T-cells in pancreatic islets. Diabetes. 2000;49(5):708-711.

51. von Herrath MG, Fujinami RS, Whitton JL. Microorganisms and autoimmunity: making the barren field fertile? Nat Rev Microbiol. 2003;1(2):151-157.

52. Nair S, Leung KC, Rawlinson WD, Naing Z, Craig ME. Enterovirus infection induces cytokine and chemokine expression in insulin-producing cells. J Med Virol. 2010;82(11):1950-1957.

53. Berg AK, Korsgren O, Frisk G. Induction of the chemokine interferon-gamma-inducible protein-10 in human pancreatic islets during enterovirus infection. Diabetologia. 2006;49(11):2697-2703.

54. Barber DL, Wherry EJ, Ahmed R. Cutting edge: rapid in vivo killing by memory CD8 $\mathrm{T}$ cells. Jimmunol. 2003;171(1):27-31.

55. In't Veld P, et al. Screening for insulitis in adult autoantibody-positive organ donors. Diabetes. 2007;56(9):2400-2404.

56. Okabe M, Ikawa M, Kominami K, Nakanishi T, Nishimune Y. 'Green mice' as a source of ubiquitous green cells. FEBS Lett. 1997;407(3):313-319.

57. Le Borgne $\mathrm{M}$, et al. The impact of negative selection on thymocyte migration in the medulla. Nat Immunol. 2009;10(8):823-830.

58. Thévenaz P, Ruttimann UE, Unser M. A pyramid approach to subpixel registration based on intensity. IEEE Trans Image Process. 1998;7(1):27-41. 